\title{
Continuous Production of Polymer Nanoparticles Using a Membrane-Based Flow Cell
}

\author{
Zhengnan Yang, Dona Foster, Ali Dhinojwala \\ Department of Polymer Science, The University of Akron, Akron, OH 44325-3909, USA
}

\begin{abstract}
We demonstrate the surfactant-free production of polymer nanoparticles using a continuous membrane-based tangential flow cell. Co-current streams of water and polymethylmethacrylate (PMMA)/acetone/water solution were separated by a porous regenerated cellulose (RC) membrane. The water concentration in the PMMA solution was adjusted so that as additional water diffused through the RC membrane, the PMMA solution composition crossed the two phase boundary to precipitate PMMA nanoparticles. The size of these nanoparticles varied with the concentration of the PMMA feed and the amount of water diffusing across the membrane. The size distribution of PMMA particles produced in a continuous flow membrane cell was much narrower than those produced by drop-wise water addition or batch dialysis precipitation of PMMA particles. A continuous production of polymer nanoparticles of high purity and narrow polydispersity are important requirements for biomedical applications such as delivering therapeutics.
\end{abstract}

Keywords: Polymer Nanoparticles, Membrane, Solvent-shifting

\section{Introduction}

Polymer nanoparticles find applications in drug delivery, medical imaging, structural colors, composites, and paints[1, 2, 3, 4, 5, 6, 7, 8, 9, 10]. Nanoparticle preparation methods include emulsion or micro-emulsion polymerization and precipitation from polymer solution $[11,12,13,14]$. In emulsion polymerization potentially toxic surfactants, initiators and oligomers yield contaminated nanoparticles unsuitable for drug and medical applications[15, 16].

Email address: ali4@uakron.edu (Ali Dhinojwala)

Preprint submitted to Journal of Colloid and Interface Science

April 12, 2017

(C) 2017. This manuscript version is made available under the Elsevier user license

http://www.elsevier.com/open-access/userlicense/1.0/ 
Nanoparticle precipitation from polymer solution has been accomplished by methods of non-solvent addition[13]. The mechanism for nanoparticle formation from solution is to transfer the solution from the stable single phase region to the unstable region of the appropriate phase diagram by nonsolvent addition or thermal shifting. For nonsolvent addition, current methods include drop-wise addition, flash precipitation and dialysis[17, 18]. Solutions of pre-synthesized contaminant-free polymers having well defined molecular weights may be dialyzed or mixed directly with nonsolvent to provide the purity needed for biomedical applications $[2,4,19,20]$. Dialysis with nonsolvent is a batch process and inherently slow. To speed up polymer nanoparticle formation, the polymer solution can be directly poured into a non-solvent (water) while it is vigorously stirred[21, 18, 22, 23, 24]. Particle size and size distribution depend on mixing kinetics as well as the initial polymer concentration and result in a significant polydispersity [25, 26].

In this work nanoparticles with controlled size and a narrow polydispersity are produced by a continuous membrane-based solvent shifting process. A membrane separates two co-current streams: polymer solution and nonsolvent. The flow cell provides a large membrane surface area to volume ratio and the concentration of water in the polymer solution is increased more homogeneously than in the flash precipitation process and much more rapidly than in the dialysis process. Our new process takes advantage of well characterized and commercially available membranes and membrane processes for easy scale-up to mass production of nanoparticles. The continuous process is readily extended to polymers precipitated by a miscible solvent/non-solvent combination such as polystyrene, polyvinylacetate, polycarbonate and polyvinylcarbazole[24]. For applications in drug delivery, hydrophobic drug molecules may be added to the polymer solution thereby directly incorporating therapeutics in the nanoparticles during the phase separation process[19].

\section{Experimental Methods}

\subsection{Materials and Solvents}

Atactic PMMA $\overline{M_{w}}=75,000 \mathrm{~g} / \mathrm{mol}(P D I \leqslant 1.06)$ was purchased from Scientific Polymer Products and dissolved in HPLC grade acetone (99.9 wt.\% purity, Sigma Aldrich) to form a solution with a concentration of $1 \cdot 10^{-4}$ $\mathrm{g} / \mathrm{g}$. The solution was stirred for 24 hours and filtered three times using a $450 \mathrm{~nm}$ Teflon filter to remove the dust particles. Millipore Ultrapure Type 
I water (18.2 $\Omega \mathrm{M}, \mathrm{pH} 6$ to 7 ) was used in all experiments. Spectra/Por ${ }^{\circledR}$ regenerated cellulose (RC) membranes, 12-14 kDa molecular weight cut-off, was purchased from Spectrum Laboratories, Inc and was used in both dialysis process and the membrane flow cell.

\subsection{Nanoparticle Production by Drop-wise Addition of Non-solvent into So- lution}

Water was added drop-wise $(0.1 \mathrm{ml} / \mathrm{s})$ into $5 \mathrm{ml}$ of $1 \cdot 10^{-4} \mathrm{~g}$ PMMA solution without stirring at $22 \circ C$, after which the particle suspension was drop cast for SEM characterization. The particle suspension was poured into $30 \mathrm{ml}$ of pure water without stirring to quench the morphology of produced PMMA nanoparticles for storage and DLS characterization. Drop-wise addition of non-solvent was also used to determine the concentration of water necessary for nanoparticles to form (cloud point). Acetone was not evaporated before light scattering measurement.

\subsection{Nanoparticle Production by Batch Dialysis}

In batch dialysis process, $5 \mathrm{ml}$ of PMMA solution was placed in the dialysis bag and dialyzed against $2 L$ of water with mild stirring at $22 \circ C$. In the initial two hours water was replaced by fresh water every 30 minutes. Then every hour the water was refreshed and the total dialysis time was 24 hours. PMMA particle suspension in water was collected for further characterization and storage.

\subsection{Nanoparticle Production by Membrane Flow Cell}

A membrane cell was designed and built to hold the RC membrane and to flow the solvent/solution on one side of the membrane and the non-solvent on the other as shown in Figure 1. A Masterflex peristaltic pump controlled the flow of the two liquids. Co-current flow was chosen in order to maintain a consistent driving force along the membrane. The flow cell was primed for 9 s before starting an experiment. The membrane rejects polymer, but allows small molecules to diffuse through the membrane. Nonsolvent (water) flux across the membrane was determined by flowing pure solvent (acetone) and pure non-solvent (water) co-currently as in the configuration shown in Figure 1 (b). Because water diffuses preferentially through the RC membrane (due to an anomalous difference in the diffusivity of water into acetone as compared to acetone into water[28]) the water flux is greater than the reverse flux of acetone into the water phase. To optimize the co-current water flux 


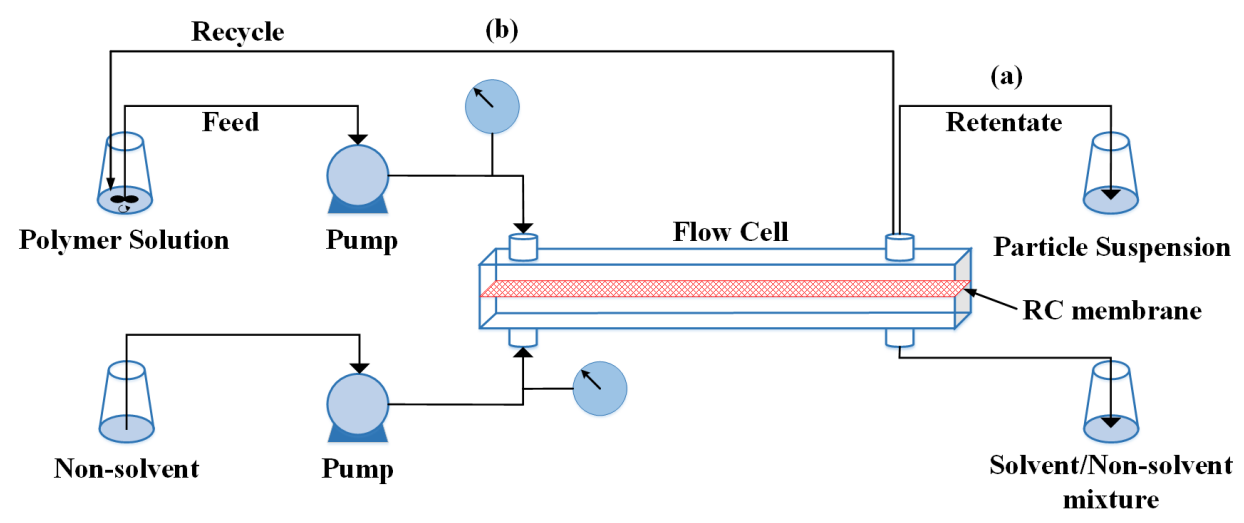

Figure 1: Schematic of a RC membrane flow cell illustrating two continuous flow configurations: (a) single pass mode and (b) recycling mode where the polymer solution is recycled back into the polymer feed solution.

relative to solvent volumes and flow cell, flow rates of $\left(F_{s}=10 \mathrm{~mL} / \mathrm{min}\right)$ for polymer solution and $\left(F_{w}=74 \mathrm{~mL} / \mathrm{min}\right)$ for water were chosen to maximize water flux for the volumes, membrane size, and pump speeds available. The weight $\%$ of water in the acetone was determined by measuring the refractive index of the mixture using a refractometer (Atago Co, NAR-3T). The refractive indices were converted to weight\% of water (Figure A.5) using the calibration curve (Appendix Figure A.7) created from standard mixtures at $22 \circ C$. By sampling the retentate at 5 minute intervals, measuring the refractive index of the mixture and comparing to the calibration curve, the weight $\%$ of water transferred into acetone was determined as a funcion of time (Appendix Figure A.6). During the 9s priming of the system there was an abrupt initial increase in water. Steady state water flux occurred after about 5 minutes. The mass flux of water across the membrane is given by the slope at steady state, approximately $f_{w}=0.4 \mathrm{wt} . \% / \mathrm{min}(y=0.40 \cdot x+2.86)$.

The amount of water needed to induce phase separation was known from drop-wise addition cloud point. However, the mass flow of water across the membrane in a single pass was not sufficient to form nanoparticles in a single pass. By spiking the initial polymer solution feed with water, we were able to adjust the feed solution in order to induce phase separation and precipitate nanoparticles in a single pass. Appendix Figure A.8 shows the average intensity of scattered light from a $C_{i}=1 \cdot 10^{-4} \mathrm{~g} / \mathrm{g}$ polymer solution as a function of $\mathrm{wt} \%$ water added. The scattered light intensity 
increased sharply at the onset water fraction for forming PMMA particles, approximately $W_{c}=18.91 w t . \%$. Feed streams were adjusted to contain $18.69 w t . \%$ water and nanoparticles were formed during a single pass through the flow cell with mass flux of water of $f_{w}=0.4 w t . \% / \mathrm{min}$ and residence time of 1.2 minutes.

Nanoparticles were produced using the flow cell operating in single pass mode as shown in Figure 1 (a). Water flux $f_{w}$ across the membrane into the polymer solution resulted in an increase in the overall mass of water in the polymer solution inducing phase separation of PMMA nanoparticles. Collected PMMA nanoparticle suspensions were drop cast for SEM measurements. For light scattering characterization and storage, each $5 \mathrm{ml}$ of collected particle suspension was poured into $30 \mathrm{ml}$ of water to quench the morphology of produced PMMA nanoparticles. Acetone was not evaporated before light scattering measurements.

\subsection{Particle Characterization}

Collected PMMA nanoparticle suspensions were directly used for SEM microscopy. For storage and light scattering characterization, each $5 \mathrm{ml}$ of collected particle suspension was stabilized with $30 \mathrm{ml}$ of water added without stirring. The vials were tightly sealed to inhibit acetone evaporation before light scattering measurements.

Dynamic light scattering (DLS) with a $633 \mathrm{~nm}$ solid-state laser $(30 \mathrm{~mW})$ was used to measure the size and distribution of PMMA nanoparticles in the collected particle suspension. Scintillation vials $(20 \mathrm{~mL})$ were washed 3 times with acetone followed by a 5 minute air plasma (Harrick Plasma, PDC$32 \mathrm{G}$ ) treatment before filling with $5 \mathrm{~mL}$ of PMMA/acetone/water mixture or PMMA nanoparticle suspension for DLS experiments. DLS was measured with the detector at a $90^{\circ}$ angle with respect to the incoming laser beam and a data collection time of $600 \mathrm{~s}$. The particle size was calculated using the CONTIN program, which can be plotted as $\Gamma G(\Gamma)$ versus $R_{h}$, where $\Gamma$ is the time scale and $G(\Gamma)$ is the characteristic line width in the correlation function $\left|g^{(1)}(\tau)\right|=\int G(\Gamma) e^{\Gamma \tau} d \Gamma$. The value of $\Gamma_{i} G\left(\Gamma_{i}\right)$ is proportional to the scattered intensity of all the i particles having the same hydrodynamic radius $R_{h}$, and the average size was calculated using the first moment[27]. As water (non-solvent) was added to the polymer solution, the cloud point was determined when the scattered intensity increased rapidly with water concentration. 
The size and morphology of the dried PMMA particles were also measured using scanning electron microscopy (SEM, JEOL-7401, JEOL Led). Samples were prepared by dropping cast PMMA/Acetone/water mixture or PMMA nanoparticle suspension directly onto a SEM stub and drying under vacuum. To minimize charging effects and heat damage under SEM, a few $\AA$ gold was sputtered onto the PMMA particles to increase the conductivity before the SEM experiments.

\section{Results and Discussion}

We compared PMMA nanoparticles precipitated by three methods designed to slowly introduce non-solvent (water) into PMMA-solvent (acetone) solution. In Table 1 size distributions of nanoparticles formed using the membrane flow cell in single pass mode (curve (c) in Figure 2) with initial water $W_{c}=18.69 w t . \%$ in the $1 \cdot 10^{-4} \mathrm{~g} / \mathrm{g}$ PMMA/acetone solution were compared to the distributions of nanoparticles formed by drop-wise addition of $18.91 w t . \%$ water into solution containing $1 \cdot 10^{-4} \mathrm{~g} / \mathrm{g}$ PMMA in acetone (curve (a) in Figure 2 ) and by dialysis of PMMA solution against water (curve (b) in Figure 2). The nanoparticles formed in the membrane flow cell have a smaller hydrodynamic radius $R_{h}$ and a narrower size distribution $(\mathrm{H}-$ WHM) compared to nanoparticles formed by other methods using the same polymer solution concentration. For a homogeneous nucleation and growth process, the size of the particles depends on the growth time after the nuclei are formed $[29,13,30]$. The membrane flow cell allows a more controlled introduction of water into the polymer solution by minimizing concentration gradients and forming nanoparticles with a narrower size distribution compared to the particles formed by direct addition of water and dialysis.

Table 1: Comparison of two PMMA nanoparticle formation methods from $1 \cdot 10^{-4} \mathrm{~g} / \mathrm{g}$ PMMA in acetone: Direct addition of water and RC Membrane flow cell.

\begin{tabular}{|c|c|c|}
\hline & Averaged Hydrodynamic Radius $R_{h}$ & Size Distribution HWHM \\
\hline Adding Water into Solution & $110.1 \pm 17.0 \mathrm{~nm}$ & $103.1 \pm 46.9 \mathrm{~nm}$ \\
\hline Bulk Dialysis & $72.8 \pm 14.3 \mathrm{~nm}$ & $24.9 \pm 1.4 \mathrm{~nm}$ \\
\hline Membrane Flow Cell & $29.2 \pm 10.3 \mathrm{~nm}$ & $19.1 \pm 12.6 \mathrm{~nm}$ \\
\hline
\end{tabular}




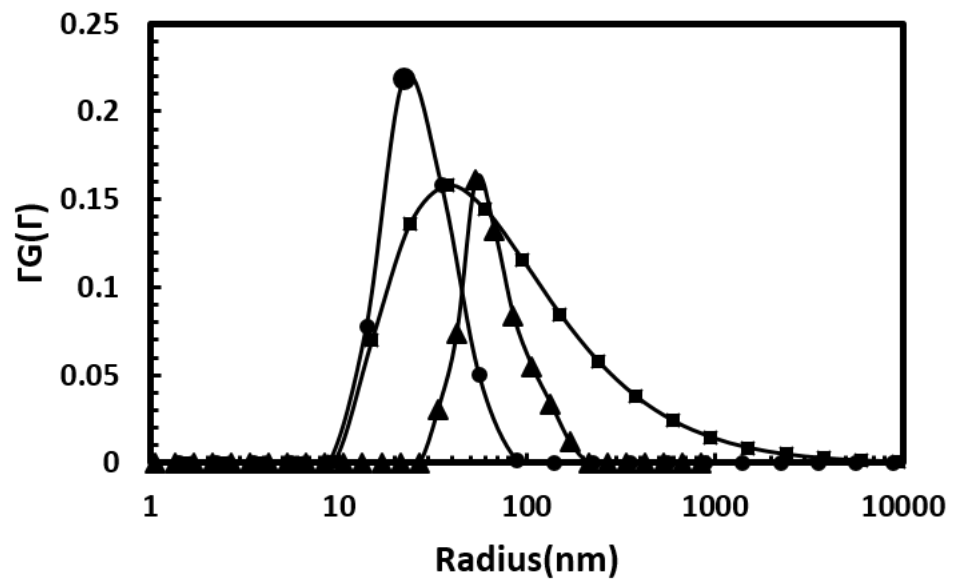

Figure 2: Particle size distribution as a plot of $\Gamma G(\Gamma)$ verse $R_{h}$. (a) •Distribution of PMMA particles produced by directly adding $18.91 w t . \%$ of water. (b) $\boldsymbol{\Delta}$ Distribution of PMMA particles produced using dialysis process. (c) • Distribution of PMMA particles produced using the continuous membrane flow cell with an initial water weight $\%$ of $W_{c}=18.69 w t . \%$. 
(a)
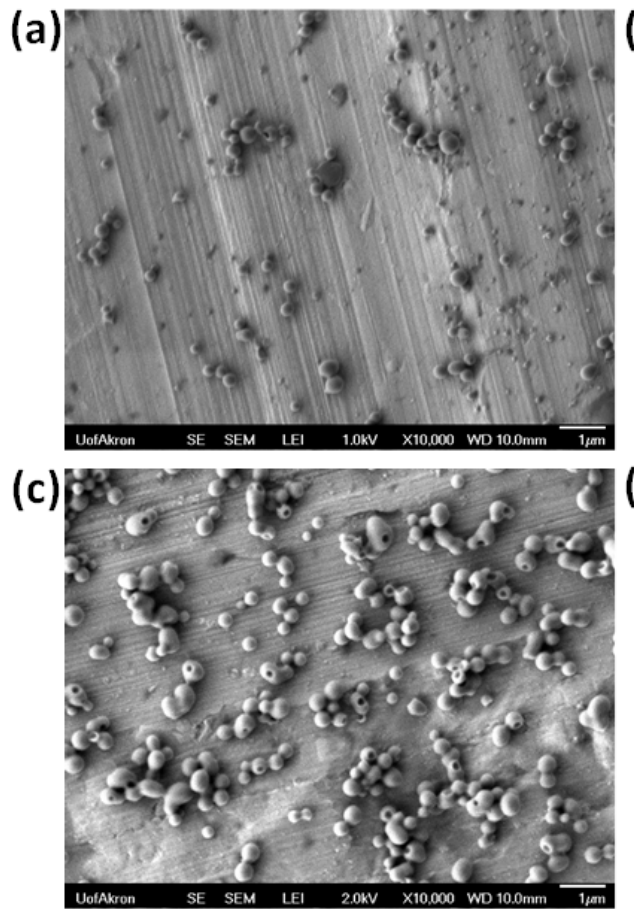

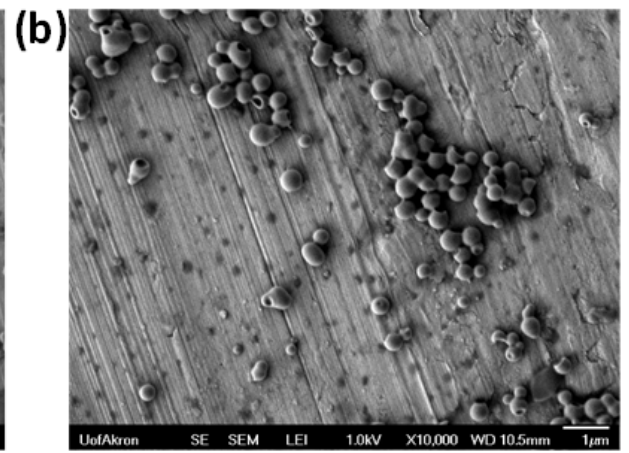

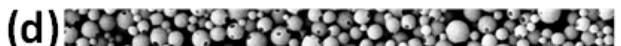

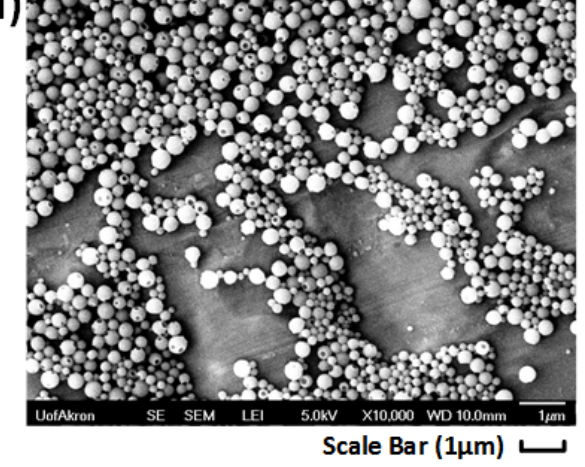

Figure 3: SEM images of dried PMMA particles produced by adding directly (a) $15.25 w t . \%$, (b) $18.03 w t . \%$, (c) $18.69 w t . \%$, and (d) $18.91 w t . \%$ of water. For weight $\% \mathrm{~s}$ less than $18.69 w t . \%$, spherical structures were formed by the phase separation caused by the faster evaporation of acetone relative to water. 
(a)

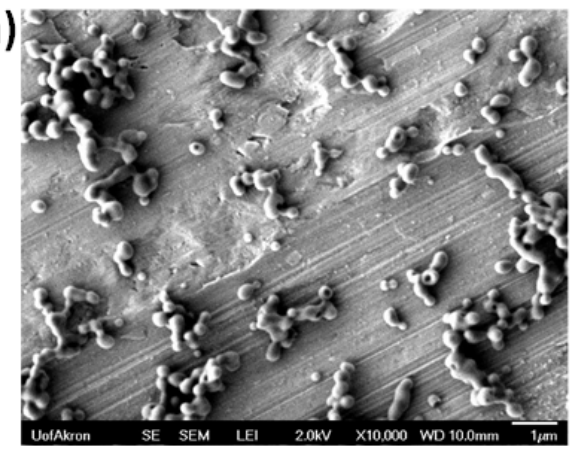

(c)

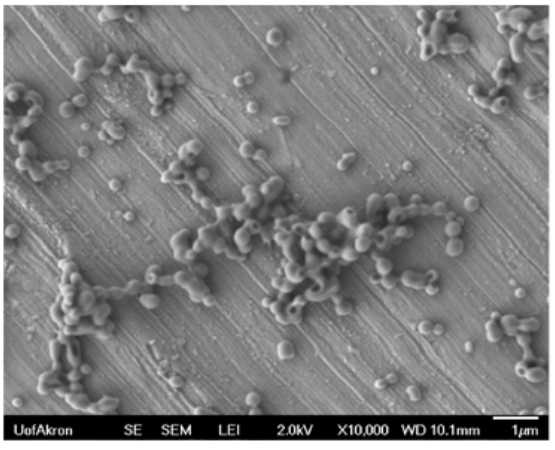

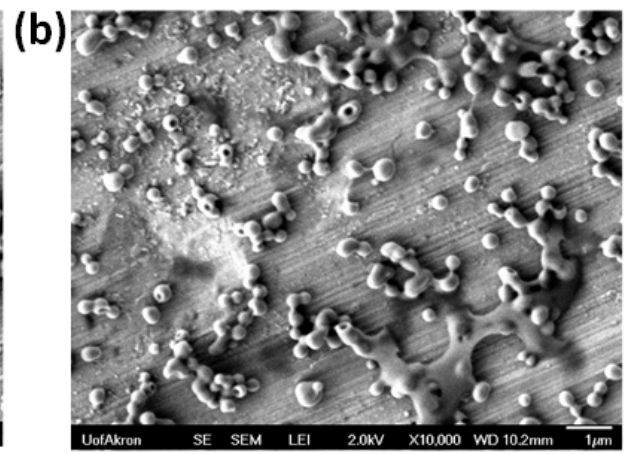

(d)

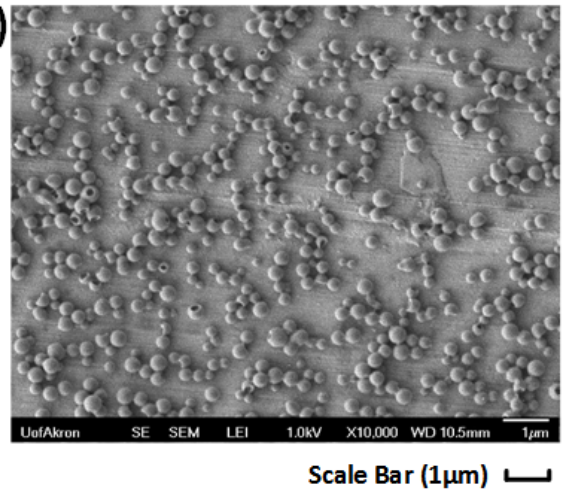

Figure 4: SEM images of dried PMMA particles produced by continuous membrane flow cell with the following initial concentration of water (a) $9.09 w t . \%$, (b) $15.25 w t . \%$, (c) $18.03 w t . \%$, and (d) $18.69 w t . \%$. For concentrations less than $18.69 w t . \%$, spherical structures were formed by the phase separation caused by faster evaporation of acetone relative to water.

We imaged the PMMA nanoparticles formed by drop-wise addition of water at $W_{c}=15.25 w t . \%, 18.03 w t . \%$, and $18.69 w t . \%$ using SEM (Figures 3 (a)-(c)). Initially, water $\left(W_{c}\right)$ was not sufficient to initiate the particle formation. We confirmed the absence of particles by DLS. Irregular shapes and sizes of nanoparticles observed in the SEM were due to a faster rate of evaporation of acetone relative to water, which led to a phase separation of the drop cast solutions. By employing an initial water concentration of $18.91 w t . \%$, the PMMA nanoparticles formed by drop-wise addition and observed under SEM were well-defined spherical PMMA particles, consistent with the results obtained from DLS.

To compare the size distributions of particles produced by the RC membrane flow cell with different amounts of water spike in the PMMA feed 
solution, we sampled the solution immediately after the first pass. Initial solutions containing less than $18.69 w t$.\% water water did not form nanoparticles within the flow cell and irregularly shaped and sized particles formed as the drop cast solutions dried (Figure 4(a)-(c)). Figure $4(\mathrm{~d})$ shows the SEM images of dried PMMA nanoparticles produced in the RC membrane flow cell at an initial concentration of $18.69 w t . \%$ water. Spherical particles were observed only when the initial water concentration and the single pass water flux were sufficiently near the cloud point, supporting our hypothesis that sufficient water had diffused across the membrane to shift the solution across the two phase boundary and generate uniform nanoparticles within the flow cell.

Both mixing effects and nanoparticle formation mechanisms have been previously studied and are reported in the literature. Aubry et al.[23] report the effects of different mixing methods on the size and size distribution of PMMA nanoparticles formed from $10^{-4} w t . \%$ PMMA in acetone solution. They consider four different mixing methods: one-shot addition of a large volume of water into PMMA-acetone solution, drop-wise addition of water into solution, drop-wise addition of PMMA-acetone solution into water and one-shot addition of a large volume of water into water spiked PMMAacetone solution. Drop-wise addition of water into PMMA solution produced nanoparticles of larger size and wider size distribution than those produced by the other three methods. Perevyazko et al.[25] also observed the same size variation among similar methods.

Two different mechanisms for the nanoparticle formation were considered to explain the apparent difference in size. For the case of drop-wise addition of water into solution, near-equilibrium phase separation induced a nucleation and growth process as the non-solvent was slowly introduced into the solution[32]. A wide size distribution was expected due to continuing nucleation such that growth times differed substantially. The other three processes produced PMMA nanoparticles as small as 70-80 nm with narrower size distributions than for drop-wise addition of water into solution. The smaller sizes and distributions were explained by a nucleation and aggregation of nanoparticles in a large volume of nonsolvent due to the supersaturated solution state[33]. Our results are consistent with those of Aubry et al. and Perevyazko et al. Drop-wise addition of water into PMMA solution yielded similar particle sizes and distributions that is, larger sized particles and wider size distributions than they obtaind by one-shot addition or drop-wise addition of solution. Drop-wise addition of water, dialysis and 
membrane flow cell processes all introduce the nonsolvent gradually, consistent with a nucleation and growth mechanism for nanoparticle formation. However dialysis and membrane flow cell both produced particles of smaller size and narrower size distribution than for drop-wise addition. The large solvent exchange area offered by the dialysis membrane minimizes the water concentration gradients so as to give similar growth times for each PMMA nanoparticle nucleated. By using a membrane flow cell, we obtained small nanoparticle sizes and size distributions (Table 1) comparable to the small particles formed during one-shot addition and drop-wise addition of solution into water reported by Aubry et al. and by Perevyazko et al. The membrane flow cell enables the continuous formation of small (20-40nm) nanoparticles while consuming considerably less water than the large volumes required to support nucleation and aggregation in the one-shot and solution addition methods.

Continuous production of polymer nanoparticles by membrane flow cell can be readily applied to form pure nanoparticles of narrow polydispersity from other polymers because the process is based on solvent-shifting across the single phase/two phase boundary. The membrane based non-solvent introduction nanoparticle formation requires a miscible solvent and non-solvent combination and solvent compatable membranes. Solvent compatable polymer and ceramic membranes are available in a wide variety of robust microand nano-porous flat sheet and hollow fiber geometries. The process can readily be adapted to the formation of amorphous polymer nanoparticles, polymer crystals, dye nanoparticles and the encapsulation of hydrophobic drugs $[2,4,19,20,7]$. Scale-up of membrane processes depends on the initial concentration of polymer in solution, non-solvent flux rates for a given membrane, membrane surface area and flow cell residence times[34] and can be tuned based on the single phase/two phase thermodynamic transition for the three component system.

\section{Conclusion}

The continuous production of surfactant-free nanoparticles having a narrower polydispersity than nanoparticles formed by direct addition of nonsolvent and dialysis method has been shown. By controlling the initial concentration of water, flow of water across the RC membrane, and the length of the flow chamber we can continuously produce nanoparticles using a single pass across the flow cell. There is need for a controlled process to make 
nanoparticles of controlled size and dispersity for applications in controlled drug delivery and therapeutics. This new continuous membrane-based design provides the required conditions for preparing pure polymer nanoparticles for biomedical applications.

\section{Acknowledgment}

The authors wish to thank the National Science Foundation for funding this research.

\section{Appendix A. Calibration of Membrane Flow Cell: Water Flux Across the Membrane and Cloud Point}

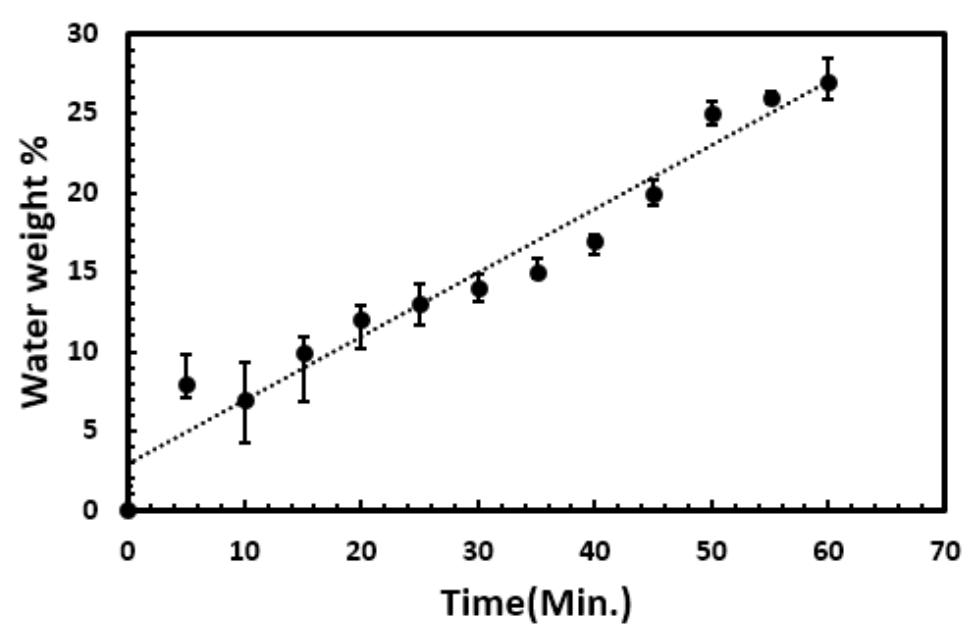

Figure A.5: The weight $\%$ of water in the acetone feed measured as a function of time measured using the flow cell configuration shown in Figure 1 (b). 


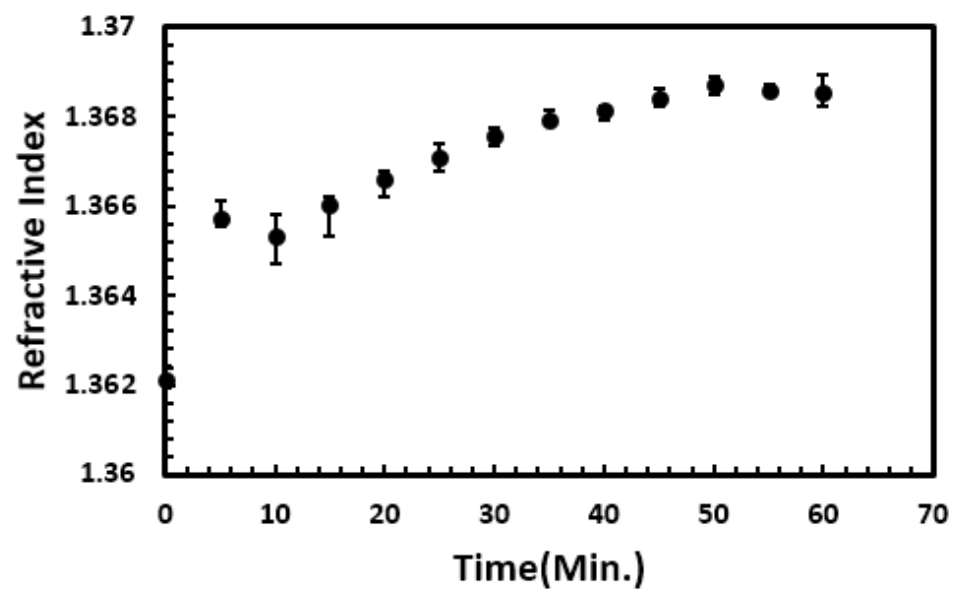

Figure A.6: Refractive index change with increase in water weight\% in acetone solution as a function of time.

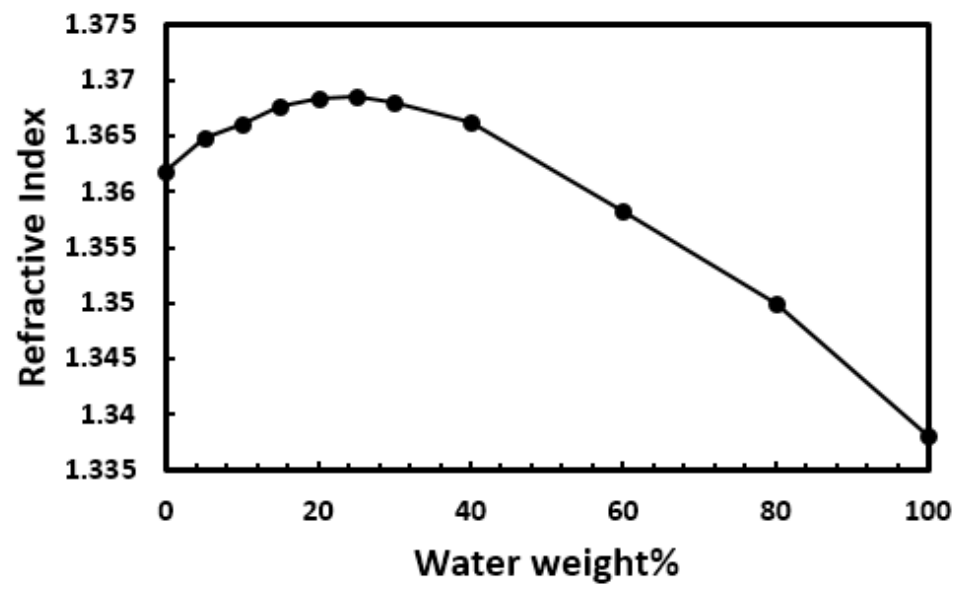

Figure A.7: Standard calibration curve of refractive index verse water mass percentage wt.\% 


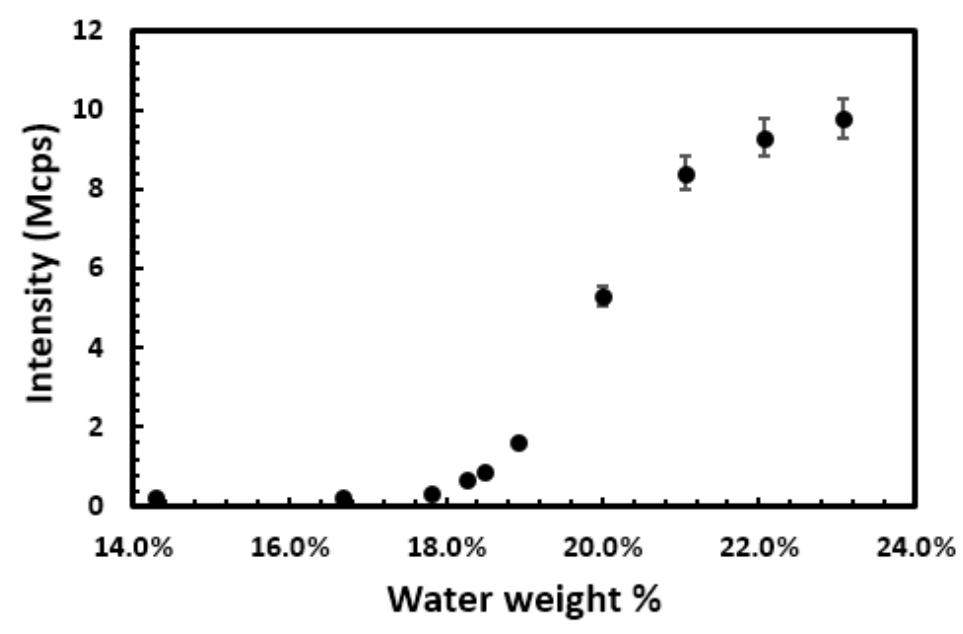

Figure A.8: Intensity of scattered light at $90^{\circ}$ configuration as a function of water weight $\%$. The inflection in the scattered light intensity corresponds to the cloud point at $W_{c}=$ $18.91 w t \%$.

[1] L. Bokobza, The reinforcement of elastomeric networks by fillers, Macromol. Mater. Eng. 289 (7) (2004) 607-621.

[2] V. Lassalle, M. L. Ferreira, Pla nano- and microparticles for drug delivery: An overview of the method and preparation, Macromol. Biosci. 7 (2007) 767-783.

[3] A. R. Parker, A vision for natural photonics, Phiolos. Trans. R. Soc. London 362 (1825) (2004) 2709-2720.

[4] Y. Masayuki, M. Mizue, Y. Noriko, O. Teruo, S. Yasuhisa, K. Kazunori, I. Shohei, Polymer micelles as novel drug carrier: adriamycin-conjugated poly (ethylene glycol)-poly (aspartic acid) block copolymer, J. Controlled Release 11 (1) (1990) 269-278.

[5] M. Lu, J. Zhou, L. Wang, W. Zhao, Y. Lu, L. Zhang, Y. Liu, Design and preparation of cross-linked polystyrene nanoparticles for elastomer reinforcement, J. Nanomater. 2010 (2010) 7-14.

[6] L. Zhang, Y. Wu, W. Yiqing, W. Yizhong, Z. Huifeng, Y. Dingsheng, H. Jianyun, The nano-reinforcing and nano-compounding technique of rubber [J], China Synthetic Rubber Industry 2 (2000) 002. 
[7] U. Bilati, E. Allemann, E. Doelker, Development of a nanoprecipitation method intended for the entrapment of hydrophilic drugs into nanoparticles, Eur. J. Pharm. Sci. 24 (2005) 67-75.

[8] J. D. Forster, H. Noh, S. F. Liew, V. Saranathan, C. F. Schreck, L. Yang, J.-G. Park, R. O. Prum, S. G. Mochrie, C. S. O'Hern, Biomimetic isotropic nanostructures for structural coloration, Adv. Mater. 22 (2627) (2010) 2939-2944.

[9] P. Buffat, J. P. Borel, Size effect on the melting temperature of gold particles, Phys. Rev. A 13 (6) (1976) 2287.

[10] Y. Matsumura, H. Maeda, A new concept for macromolecular therapeutics in cancer chemotherapy: mechanism of tumoritropic accumulation of proteins and the antitumor agent smancs, Cancer Res. 46 (12 Part 1) (1986) 6387-6392.

[11] J. P. Rao, K. E. Geckeler, Polymer nanoparticles: Preparation techniques and size-control parameters, Prog. Polym. Sci. 36 (2011) 887913.

[12] H. Fessi, F. Puisieux, J. P. Devissaguet, S. Ammoury, N.; Benita, Nanocapsule formation by interfacial polymer deposition following solvent displacement, Int. J. Pharm. 55 (1989) R1-R4.

[13] G. Francois, J. L. Katz, Nanoparticles and nanocapsules created using the ouzo effect: spontaneous emulisification as an alternative to ultrasonic and high-shear devices., Chemphyschem 6 (2) (2005) 209-216.

[14] K. Kataoka, A. Harada, Y. Nagasaki, Block copolymer micelles for drug delivery: design, characterization and biological significance, Adv. Drug Delivery Rev. 47 (1) (2001) 113-131.

[15] G. Odian, Principles of polymerization, Vol. 3, Wiley-Interscience New York, 2004.

[16] K. Tauer, R. Deckwer, I. Kuhn, C. Schellenberg, A comprehensive experimental study of surfactant-free emulsion polymerization of styrene, Colloid and Polym. Sci. 277 (7) (1999) 607-626. 
[17] C. Zhang, J. W. Chuang, R. D. Priestley, Dialysis nanoprecipitation of polystyrene nanoparticles, Macromol. Rapid Commun. 33 (2012) 17981803.

[18] S. Schubert, J. Joseph T. Delaney, U. S. Schubertb, Nanoprecipitation and nanoformation of polymer: from history to powerful possibilities beyond poly(lactic acid), Soft Matter 7 (2010) 1581-1588.

[19] D. M. Zarkadas, K. K. Sirkar, Antisolvent crystallization in porous hollow fiber devices, Chem. Eng. Sci. 61 (15) (2006) 5030-5048.

[20] A. Dalpiaz, E. Vighi, B. Pavan, E. Leo, Fabricaiton via a nonaqueous nanoprecipitation method, characterization and in vitro biological behavior of n6-cyclopentyladenosine-loaded nanoparticles, J. Pharm. Sci. 98 (11) (2009) 4272-4284.

[21] A. Vollrath, A. Schallon, C. Pietsch, S. Schubert, T. Nomoto, Y. Matsumoto, K. Kataoka, U. S. Schubert, A toolbox of differently sized and labeled pmma nanoparticles for cellular uptake investigations, Soft Matter 9 (1) (2013) 99-108.

[22] C. Zhang, V. J. Pansare, R. K. Prud'homme, R. D. Priestley, Flash nanoprecipitation of polystyrene nanoparticles, Soft Matter 8 (2012) 8693.

[23] J. Aubry, F. Ganachaud, J.-P. C. Added, B. Cabane, Nanoprecipitation of polymethylmethacrylate by solvent shifting: 1 . boundaries, Langmuir 25 (2009) 1970-1979.

[24] S. Hornig, T. Heinze, C. R. Becer, U. S. Schubertb, Synthetic polymer nanoparticles by nanoprecipitation, J. Mater. Chem. 19 (2009) 3838.

[25] I. Y. Perevyazko, A. Vollrath, C. Pietsch, S. Schubert, G. M. Pavlov, U. S. Schubert, Nanoparecipitation of poly(methyl methacrylate)-based nanoparticles: Effect of the molar mass and polymer behavior, Polym. Chem. 50 (2012) 2906-2913.

[26] I. Perevazko, A. Vollrath, S. Hornig, G. M. Pavlov, U. S. Schubert, Characterization of poly(methyl methacrylate) nanoparticles prepared by nanoprecipitation using analytical ultracentrifugation, dynamic light 
scattering, and scanning electron microscopy, Polym. Chem. 48 (2010) 3924-3931.

[27] F. R. Hallett, Particle size analysis by dynamic light scattering, Food Res. Int. 27 (1994) 195-198.

[28] A. I. Toryanik, V. N. Taranenko, Molecular mobility and structure in water-acetone mixture, J. Struct. Chem. 28 (1998) 714.

[29] S. A. Vitale, J. L. Katz, Liquid droplet dispersions formed by homogeneous liquid-liquid nucleation:" the ouze effect", Langmuir 19 (2003) 4105-4110.

[30] N. T. Thanh, N. Maclean, S. Mahiddine, Mechanisms of nucleation and growth of nanoparticles in solution, Chem. Rev. 114 (2014) 7610-7630.

[31] D. Chen, D. Singh, K. K. Sirkar, Continuous synthesis of polymer-coated drug pparticle by pours hollow fiber membrane-based antisolvent crystallization, Langmuir 31 (1) (2015) 432-441.

[32] P. Debenedetti, Metastable Liquids, Princeton University Press: Princeton, NJ, 1996.

[33] J. A. Dirksen, T. A. Ring, Fundamentals of crystallization: Kinetic effects on particle size distributions and morphology, Chem. Eng. Sci. 46 (1991) 2389.

[34] I. Pinnau, B. D. Freeman (Eds.), Membrane Formation and Modification, American Chemical Society, 2000. 


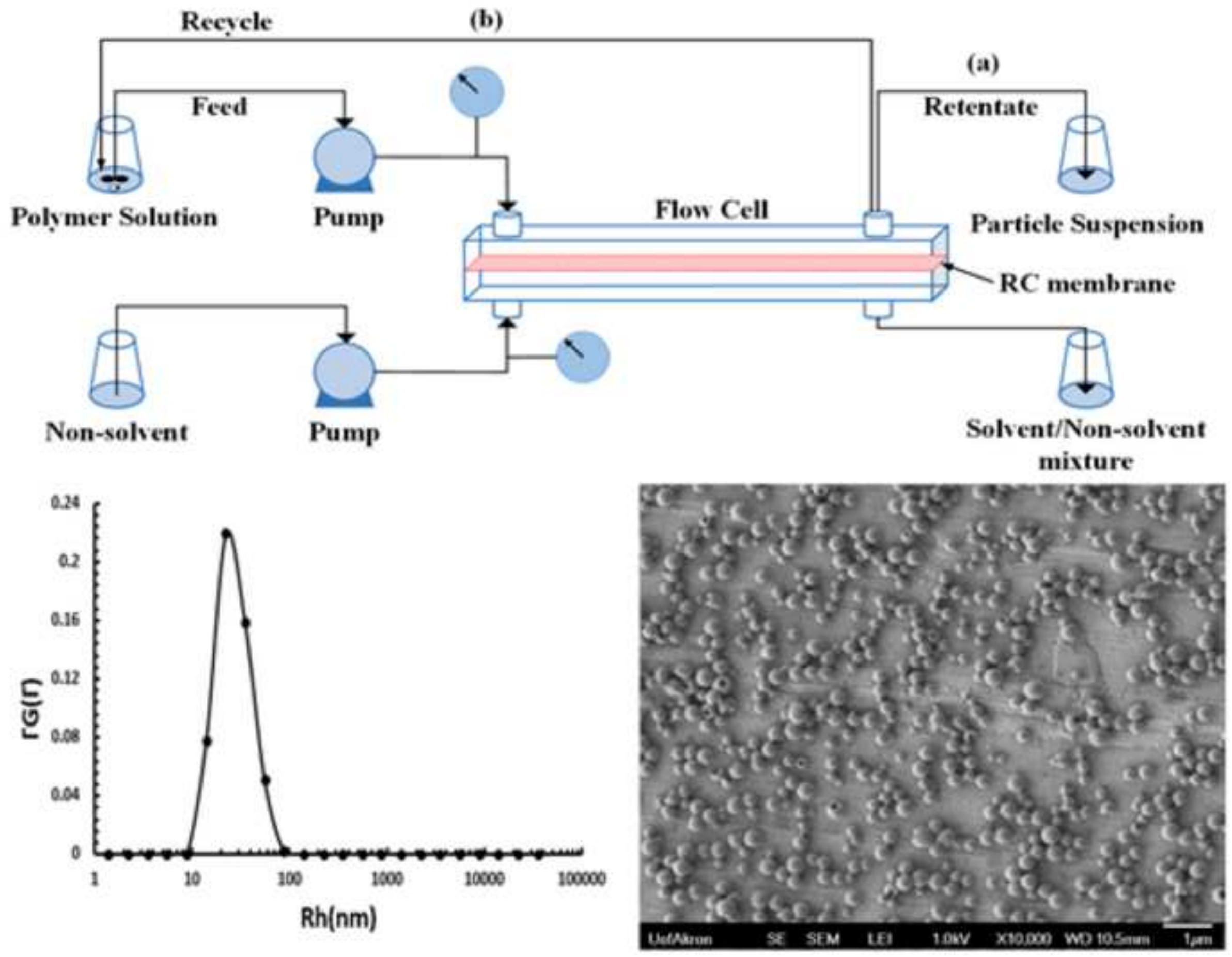

